Einführung in das Schwerpunktthema

\section{Zwischen Vision und Machbarkeit}

$\mathrm{D}$

Von Gerd Scholl

er Gedanke ist so simpel wie überzeugend: Güter werden in der Regel nicht um ihrer selbst willen gekauft und genutzt, sondern weil sie uns bestimmte Dienste leisten. Nimmt man an, dass ,immaterielle“ Dienstleistungen weniger ressourcenintensiv sind als ,,materielle" Produkte, so liegt es nahe, Bedürfnisse zusehends über Serviceangebote zu befriedigen. Geht der positive Umwelteffekt mit einem hohen Kundennutzen einher, spricht man von öko-effizienten Dienstleistungen.

Dieser Gedankengang lässt sich operationalisieren, indem man die Nutzungsintensität als das Verhältnis von abgegebener Leistung (als Indikator für das Maß der Bedürfnisbefriedigung) zu verwendetem Produktinput (als Indikator für den Ressourcenverzehr) definiert. Zahlreiche Ansätze tun sich auf (siehe Tabelle 1 auf S. 11):

- Das produktimmanente multifunktionale Design als Bündelung vormals separater Funktionen in einem Gerät steht für eine Erhöhung der Nutzungsintensität.

- Bei produktbegleitenden Servicekonzepten wie beispielsweise Reparaturstrategien geht es eher um eine Verlängerung der Nutzungszeiten beziehungsweise um die Realisierung der optimalen Nutzungsdauer.

- So genannte nutzungsorientierte oder auch produktersetzende Dienstleistungen basieren auf einem Wechsel der Verfïgungsrechte vom Kunden auf den Dienstleistungsanbieter: Produkte werden dann nicht mehr eigens erworben, sondern nur noch bei Bedarf geliehen oder gemietet.

- Bei ergebnisorientierten Dienstleistungen geht es um die sachgutunabhängige Erfuillung des Kundennutzens. Die Auswahl des zur Bedürfnisbefriedigung nötigen Produkt-DienstleistungBündels obliegt dann allein dem Anbieter.

\section{- Diskussionslinien}

Das Thema der optimalen Nutzungsdauer von Produkten wird bereits seit Ende der Siebzigerjahre diskutiert (1). Zentrale Beiträge zur Konzeptionierung und praktischen Umsetzung von Konzepten zur längeren und auch intensiveren Güternutzung gingen von den Aktivitäten des Instituts für Produktdauerforschung aus. Hier wird den bisher linearen Verständnissen des Wirtschaf- tens eine zyklische Betrachtungsweise gegenüber gestellt, bei der Stoffströme über den gesamten Produktlebenszyklus optimiert werden. Die Vision der nutzungsorientierten Dienstleistungsgesellschaft verspricht eine dreifache Dividende: Ressourcenschonung, hohe Dienstleistungs- und damit Arbeitsintensität sowie regionalisiertes Wirtschaften.

Nach einer Reihe weiterer Konzeptionierungsarbeiten und einer Vielzahl von Beispielsammlungen konzentriert sich die Debatte nun zunehmend auf die Analyse der konkreten Umsetzungsbedingungen solcher Strategien. Dazu gehören, insbesondere im Falle konsumnaher Ansätze, die Identifikation relevanter Zielgruppen oder die Berïcksichtigung von Konsum- und Lebensstilen. Des Weiteren liefern branchenbezogene Untersuchungen Erkenntnisse bezüglich hemmender und fördernder Rahmenbedingungen sowie angebotsseitiger Diffusionsprozesse.

Die oft von Visionen getragene Euphorie der Anfangsjahre ist allmählich von einer differenzierteren Betrachtungsweise der Machbarkeit abgelöst worden, die sowohl Chancen als auch Grenzen dienstleistungsorientierter Transformationsprozesse aufzeigt. Dieser Perspektivwechsel findet sich auch in der Förderpolitik des Bundes: So hat das Bundesministerium für Bildung und Forschung einen zweiteiligen Förderschwerpunkt mit dem Titel „Möglichkeiten und Grenzen von neuen Produktnutzungsstrategien" aufgelegt, der sich regionalen und bedürfnisfeldorientierten Ansätzen widmet (2).

\section{- Die Beiträge im Überblick}

Die Beiträge des Schwerpunktthemas schließen hieran an. Der Artikel von Hirscbl/Konrad/Scholl gibt einen Überblick über mögliche praktische Ansätze für öko-effiziente Dienstleistungen, charakterisiert Transformationsprozesse hin zum dienstleistungsbasierten Wirtschaften, identifiziert unterschiedliche Zielgruppen (Nutzungstypen) für Servicekonzepte und quantifiziert beispielhaft die Umweltwirkungen. Scherborn betont, dass die Dienstleistungsgesellschaft einen anderen Produktivitätsbegriff erfordert, in dem die Qualität eine größere Rolle spielen muss. Der Beitrag der Menschen, die die Dienstleistung erstellen, rückt damit in den Vordergrund, steht jedoch nach wie vor institutionellen Blockaden gegenüber. Schrader macht dann am konkreten Beispiel von CarSharing und Wasch-Services deutlich, dass mit Blick auf die Konsumentenakzeptanz eigentumsersetzender Dienstleistungen nicht nur die Veränderung der Verfügungsrechte, sondern auch die Verschiebung in den Verfügungspflichten von großer Bedeutung ist. Der Bereich Mobilität dient Frick/Knie/Reindl zur Illustrierung der Beobachtung, dass weniger der Mangel an innovativen Konzepten, sondern vielmehr die Trägheit der Umsetzungsakteure der Diffusion von ressourcenschonenden Servicelösungen im Wege steht. $A x$ / Bierter identifizieren hier regionale Strategien der Kreislaufschließung auf hohem Wertschöpfungsniveau als einen möglichen Ausweg, um das Innovationspotenzial der Akteure zu mobilisieren. Zundel schließt den Schwerpunkt mit einigen Thesen ab, in denen er unter anderem laufende Trends aufzeigt, welche die Verbreitung von Strategien der intensiveren und längeren Güternutzung unterstïtzen können. Genannt seien die zunehmende Dienstleistungsorientierung und Digitalisierung der Wirtschaft, die wachsende Flexibilisierung und Individualisierung von Lebens- und Arbeitsstilen oder der demografische Wandel der Gesellschaft.

Es wird die zentrale Herausforderung bei der zukünftigen Erforschung öko-effizienter Dienstleistungskonzepte sein, an solche Entwicklungen anzuschließen.

\section{Anmerkungen}

(1) Für Literaturhinweise siehe den Literaturkasten auf S.25: Eine Darstellung des Forschungsstands findet sich in Scholl, Gerd Ulrich/ Hirschl, Bernd/Tibitanzl, Frank: Produkte länger und intensiver nutzen. Zur Systematisierung und ökologischen Beurteilung alternativer Nutzungskonzepte, IOW-Schriftenreihe Nr. 134/98, Berlin 1998.

(2) Im Internet unter www.bmbf.de/foerde01/ forschung/3-2-2-1-6.htm und www.bmbf.de/foerde01/ forschung /3-2-2-3-0.htm.

\section{Der Autor}

Gerd Scholl ist Leiter des Forschungsfeldes Ökologische Produktpolitik im Institut für ökologische Wirtschaftsforschung.

Kontakt: IÖW, Potsdamer Str. 105, 10785 Berlin, Tel. 030/ 884594-20, Fax 030/ 8825439, E-mail: gerd.scholl@ioew.de 
(c) 20I0 Authors; licensee IÖW and oekom verlag. This is an article distributed under the terms of the Creative Commons Attribution Non-Commercial No Derivates License (http://creativecommons.org/licenses/by-nc-nd/3.o/), which permits unrestricted use, distribution, and reproduction in any medium, provided the original work is properly cited. 